\title{
Évaluer les effets des dispositifs ludiques destinés aux enfants en visite familiale
}

Assess the effects of edutaining projects aimed at children visiting with their families

\section{Claire Barbieux}

\section{(2) OpenEdition}

\section{Journals}

Édition électronique

URL : http://journals.openedition.org/ocim/870

DOI : $10.4000 /$ ocim. 870

ISSN : 2108-646X

Éditeur

OCIM

Édition imprimée

Date de publication : 1 mai 2011

Pagination : 19-26

ISSN : 0994-1908

Référence électronique

Claire Barbieux, "Évaluer les effets des dispositifs ludiques destinés aux enfants en visite familiale », La Lettre de l'OCIM [En ligne], 135 | 2011, mis en ligne le 01 mai 2013, consulté le 01 mai 2019. URL http://journals.openedition.org/ocim/870; DOI : 10.4000/ocim.870 
Cette méthode d'évaluation qualitative a été élaborée dans le cadre d'un mémoire de Master 2 "Médiations de la culture et du patrimoine à Avignon » sous la direction de Daniel Jacobi. La recherche a porté sur deux jeux spécifiques proposés dans deux musées du Parc naturel régional des Vosges du Nord : le musée de l'Image populaire à Pfaffenhoffen et le musée de la Bataille du 6 août 1870 à Woerth. Ce terrain d'étude est assez singulier : il s'agit de deux petits musées, situés en territoire rural et faiblement fréquentés. Ce qui nous a obligés à interroger un échantillon non aléatoire de jeunes visiteurs avertis car recrutés à cet effet. C'est pour cette raison que nous avons choisi de ne pas commenter de manière approfondie les résultats obtenus qui ne peuvent dans ces conditions prétendre avoir une portée générale.

\section{Que signifie jouer au musée en famille ?}

\section{Qu'est-ce que le jeu?}

Le champ lexico-sémantique du mot jeu est fréquemment utilisé dans les musées pour désigner de nombreuses activités pédagogiques impliquant une démarche active des enfants. Il convient donc au préalable de redonner sa spécificité au jeu pour pouvoir déterminer ce qui relève réellement du jeu parmi tous les différents supports pédagogiques habituellement destinés aux enfants dans les musées.

Le jeu est une notion polysémique. On peut cependant d'emblée faire une première distinction entre le jeu comme activité subjective de jouer, le jeu comme structure (c'est-à-dire un ensemble de règles) et le jeu comme matériel (c'est-à-dire l'ensemble des objets nécessaires pour jouer). En confrontant les théories du jeu dans diverses disciplines (sociologie, philosophie, sciences de la communication), nous retiendrons particulièrement l'importance du cadre ludique : le jeu est une structure ferme et réglée, et les joueurs ne peuvent jouer qu'en connaissant et respectant un code. Nous pouvons ainsi définir le jeu à la fois par son cadrage légalitaire et par le cadrage de la modalisation.

Le terme légaliberté a été proposé par Colas Duflos pour désigner le lien spécifique qui s'établit dans le jeu entre la règle et la liberté : «Le jeu est l'invention d'une liberté dans et par une légalité » (Duflos, 1997 : 57). Cette liberté réglée se traduit par le degré de liberté dans les décisions prises par le joueur à l'intérieur de la marge de manœuvre laissée par la règle du jeu. L'information dont dispose le joueur n'est jamais totalement complète et ce dernier ne peut donc être totalement assuré de l'efficacité de ses décisions. Ainsi, selon la formule de Pierre Parlebas, citée par Henriot (1989 : 239), «chaque joueur est un centre de décision et d'action dont les comportements sont porteurs d'imprévus».

Les concepts de "modalisation" (Goffman, 1991 : 50-54), ou de «métaphore " (Henriot, 1989 : 260) correspondent au fait qu' "on ne joue jamais qu'au second degré ». Le jeu opère une transposition par rapport à la réalité et instaure une mise à distance du joueur à l'égard de son jeu. C'est aussi ce que souligne Gregory Bateson (1977 : 209) quand il explique que la méta-communication accompagne le jeu dans son déroulement et véhicule à travers des signaux le message : «ceci est un jeu ».

\section{L'investissement pédagogique du jeu au musée}

En conférant une dimension éducative à des activités ludiques, les musées élaborent les jeux correspondant aux objectifs pédagogiques qu'ils ont privilégiés. Ces objectifs, plus ou moins explicites, transforment le jeu selon des buts de nature différente puisque cherchant, non pas à distraire mais à provoquer un apprentissage. Les effets attendus par les institutions muséales peuvent être variés, de la régulation du parcours des enfants dans le musée à des objectifs plus spécifiquement éducatifs : développer les capacités perceptivo-cognitives, le questionnement, la quête d'information, la compréhension des objets... D'autres effets ne sont pas toujours objectivement formulés tels que faciliter l'acquisition des règles et des conduites à adopter dans le musée, ou stimuler la convivialité, les interactions familiales.

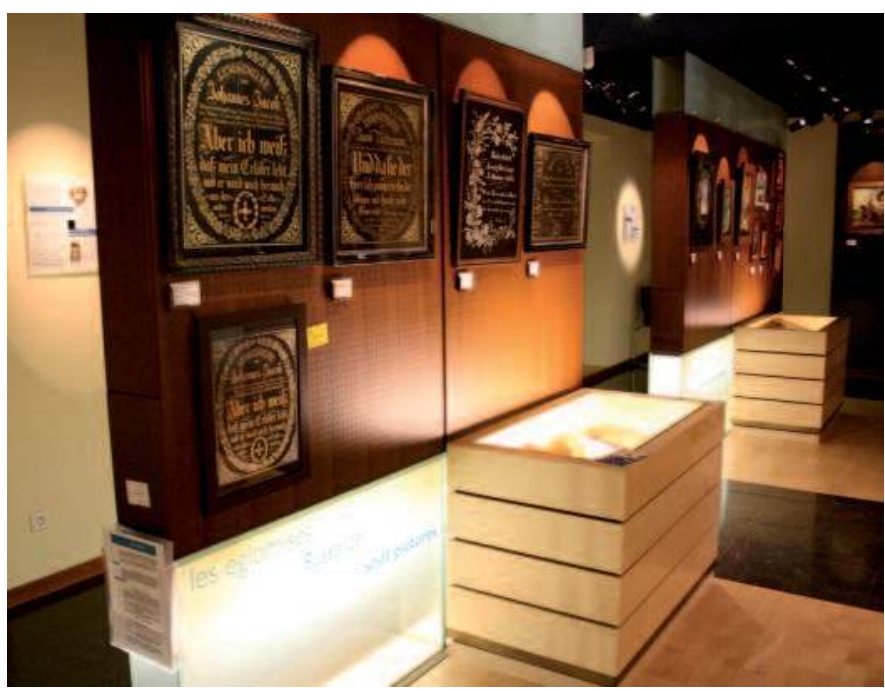

Intérieur du musée de I'Image populaire à Pfaffenhoffen, rez-de-chaussée, section des églomisés (C) Claire Barbieux 
Mais, ainsi que le souligne Gilles Bougère (2005), le processus de formalisation éducative du jeu ne passe pas seulement par la création d'un matériel ludique qui inclut une finalité pédagogique. On peut également analyser le niveau d'intervention des parents accompagnant l'enfant. Ou encore, dans le jeu, la nature de ce que l'enfant peut ou doit faire pour comprendre, s'informer ou agir. Ces trois niveaux doivent être pris en compte dans la mise en place d'une évaluation des effets du jeu pédagogique sur la visite des enfants en famille.

Les recherches menées par Anne Jonchéry (2008: 13) sur les bénéfices recherchés par les familles lors de la venue au musée concluent que les familles " investissent l'équipement muséal de fortes motivations éducatives [...] Les objectifs recherchés (réussite scolaire, intégration sociale, épanouissement personnel) révèlent combien ces usages s'inscrivent dans les fonctions de la famille ». La visite familiale vise aussi à faire plaisir à l'enfant et satisfaire ses intérêts et audelà favoriser le développement personnel et la construction identitaire de l'enfant.

Au regard de ces logiques de visite, il semble que la pratique du jeu cristallise l'ensemble des motivations qui provoquent la visite du musée en famille. Le concept d'expérience optimale (flow) développé par Csikszentmihalyi et Robinson (1975) nous apprend que le jeu est susceptible de procurer un état de bien-être quand il offre des opportunités d'action qui correspondent aux capacités du sujet. Les analyses développées dans le domaine de la psychologie de l'enfant (Piaget, 1945 ; Vygostki, 1967) et de la sociologie cognitive (Mead, 1934) considèrent que le jeu est lui-même porteur d'effets liés au développement de l'enfant et à la construction sociale et identitaire de ce dernier.

À cet effet diffus d'éducation qui accompagne potentiellement le jeu, s'ajoutent les intentions spécifiquement éducatives investies dans la pratique du jeu au musée. En tentant de concilier développement de l'enfant, plaisir et éducation à travers le support éducatif du jeu, c'est bien une réponse aux motivations divergentes de la famille contemporaine que les musées proposent, même si rien n'assure la résolution de ces tensions.

Si la contribution du jeu au développement de l'enfant est difficilement mesurable ainsi que tend à le montrer par exemple une étude relativement récente (Power, 2000), des objectifs éducatifs précis, la socialisation des enfants ainsi que le plaisir qu'ils éprouvent à travers le jeu peuvent faire l'objet d'une évaluation.

\section{La mise en forme ludique}

Faire du jeu un outil d'apprentissage au musée ne revient pas seulement à l'investir d'objectifs éducatifs, mais suppose également l'aménagement plus ou moins important du jeu dans sa forme. Le jeu au musée est une construction à la fois marquée par un projet éducatif, et caractérisée par un contrôle exercé au niveau de ses dispositifs pratiques et concrets (règles et matériel).

Le jeu en famille dans un musée échappe au principal reproche adressé au jeu dans le cadre scolaire : celui d'imposer le jeu aux élèves sans leur laisser le choix d'entrer dans le jeu. Cependant, des tensions persistent entre deux facettes antagonistes : jouer et/ou apprendre. Le recours au jeu comme moyen d'apprentissage revient à faire des choix entre un certain nombre de caractéristiques qui opposent jeu et éducation. Le caractère aléatoire du processus ludique peut sembler, en effet, entrer en tension avec un projet éducatif qui poursuit des objectifs d'apprentissage (Bougère, 2005). Les finalités des actions attendues par les concepteurs des jeux et celles recherchées par les enfants pratiquant les jeux peuvent aussi paraître éloignées : les enfants jouent d'abord pour le plaisir qu'ils vont en retirer et non pas pour optimiser un quelconque apprentissage.

Considérant la suite de décisions à prendre au cours du jeu, Colas Duflos (1997 : 58) remarque que le jeu est, du point de vue pédagogique, l'apprentissage et l'expérience d'une liberté ludique dans un monde quotidien souvent semé d'interdits. De plus, l'ensemble de ces décisions s'opérant dans l'incertitude, le jeu est affirmation de soi (ou "prise de risque " selon Henriot) et apprentissage de la maitrise de l'aléatoire. Bougère (2005) rappelle aussi l'intérêt de l'analyse de Bruner (1983) qui considère le jeu comme un lieu privilégié d'expériences car les actions entreprises par les enfants sont sans conséquence en dehors du cadre ludique. Les méthodes actives d'apprentissage, initiées au début du XXe siècle par le courant de l'Éducation Nouvelle, considèrent que les enfants mémorisent mieux des connaissances ou des apprentissages lorsqu'ils y contribuent. Certains initiateurs de ces méthodes (Maria Montessori, Ovide Decroly et Édouard Claparède) ont préconisé de recourir au jeu - qu'ils ont sans aucun doute contribué à réhabiliter - parce que, à leurs yeux, il offre l'avantage d'induire la participation des enfants à l'activité.

Le jeu proposé dans le musée s'efforce donc d'associer les contraintes de la "mise en forme ludique» et de la «mise en forme éducative » de manière plus 
ou moins équilibrée. C'est cette rencontre entre jeu et éducation qu'il convient aussi d'interroger à travers une évaluation.

\section{Mesurer les effets du jeu sur la visite des enfants en famille}

Pour saisir les effets particuliers d'un jeu en famille, on ne peut se contenter d'observer le parcours des enfants lors de la visite ou de tester ce que les enfants ont retenu une fois le jeu terminé. La pratique d'un jeu en famille est une expérience collective qui suscite de nombreux commentaires aussi bien par les enfants que par les adultes qui les accompagnent. Prendre en compte les échanges au sein de la famille est nécessaire pour pouvoir mesurer les difficultés rencontrées, le plaisir éprouvé et les apprentissages mobilisés au cours du jeu.

La collecte des données s'est faite à l'aide de deux outils complémentaires :

- une observation suivie des familles pratiquant le jeu à l'aide de la vidéo,

- un questionnaire administré aux enfants à l'issue de la visite et visant à évaluer le degré d'observation des règles, la compréhension du contenu du jeu, la socialisation des enfants et leur appropriation de l'espace du musée.

\section{Les effets du jeu au cours de la visite}

Les réactions des enfants et de leurs parents ont été enregistrées à l'aide d'une caméra. En cas de séparation des membres du groupe familial, c'est toujours le même enfant qui a été isolé et observé. Le recours à l'observation suivie filmée a été retenu dans le but de tenir compte des interventions verbales et non verbales des groupes familiaux, ainsi que du nombre de consultations des éléments du jeu et des unités d'exposition.

Par l'observation des comportements des visiteurs, on a accès à la durée de chacune des étapes du jeu et au nombre de consultations (lectures ou observations) des éléments du jeu et des unités d'exposition sur lesquelles il porte. Le but est d'étudier les effets du jeu en termes de focalisation de l'attention des enfants et de l'intérêt des enfants au cours du jeu. L'élaboration de la méthode d'analyse des interactions familiales s'est appuyée sur les études existantes menées dans les musées de sciences. Nous avons cherché à croiser à la fois la valeur sémio-cognitive des conversations des visiteurs (Borun, Chambers, Cleghorn, 1996 ; Niquette, 1994 ; Dufresne-Tassé, 1995) et le rôle joué par la sociabilité dans l'apprentissage (Peignoux, Lafon, Vareille, 2000, La muséologie des sciences et ses publics : 159-180 ; Cordier, Serre, 2000, idem : 259-280).

La relation entre le contenu du jeu, l'enfant et son groupe familial est considérée selon deux dimensions différentes : la coéducation et le fonctionnement psychologique des enfants. Les échanges pris en compte concernent toujours les réactions d'un seul des enfants du groupe familial et l'ensemble des interventions familiales qui lui sont destinées. L'analyse tient compte du type de commentaire et du nombre de commentaires, ainsi que des différentes étapes du jeu pendant lesquelles ils surviennent. Nous avons également catégorisé les interventions au sein des groupes familiaux selon la classification présentée page suivante.

La motivation intrinsèque des enfants à participer au jeu peut ainsi être repérée au niveau des manifestations de plaisir des enfants, mais aussi à travers la part représentée par les interactions des parents visant à entretenir l'adhésion des enfants. Si les parents n'ont pas vraiment besoin d'encourager les enfants à jouer par leur présence affective, nous pouvons supposer que les enfants adhèrent spontanément à l'activité.

Une proportion élevée d’interventions organisationnelles peut suggérer un effort de coordination important déployé par les membres des groupes familiaux pour s'orienter dans le musée ou s'organiser matériellement avec les éléments du jeu mis à disposition.

La présence même faible d'une métacommunication sur le jeu s'est révélée un indicateur pertinent d'un cadrage secondaire mal perçu ou posant question aux parents et à leurs enfants. Ainsi, dans le cas du jeu du musée de l'Image populaire à Pfaffenhoffen, des images en fac-similé accrochées pour le jeu à proximité des images originales des collections entraînaient une certaine confusion parmi les familles.

Une part notable des interventions de coéducation destinées à montrer et dire aux enfants ce qu'il faut regarder peut traduire un souci de la réussite du jeu chez les parents, mais peut aussi être considérées comme des moments d'éducation. Étudier la proportion représentée par les interactions visant à assister les enfants à la lecture ou à l'écriture permet de mieux percevoir les implications d'un dispositif tel qu'un livret-jeu. Une forte mobilisation des familles autour du déchiffrement des textes et de l'écriture est autant de temps et d'énergie qui ne sont pas consacrés à regarder ou observer les œuvres par exemple. L'expression de difficultés ou des demandes 


\begin{tabular}{|c|c|}
\hline Les interventions de coéducation & Les interventions des enfants \\
\hline $\begin{array}{l}\text { 1. Susciter et entraîner l'adhésion envers les exigences de la } \\
\text { situation (encourager, féliciter, s'assurer que tout va bien) }\end{array}$ & 1. Manifester un plaisir lié au jeu \\
\hline $\begin{array}{l}\text { 2. Focaliser l'attention par la parole ou le geste } \\
\text { (dire quoi regarder, aider à l'observation par des } \\
\text { interventions verbales ou non verbales) }\end{array}$ & 2. Montrer ou attirer l'attention \\
\hline 3. Décrire ; comparer & $\begin{array}{l}\text { 3. Décrire, constater; comparer les indices du jeu et } \\
\text { les objets des collections }\end{array}$ \\
\hline $\begin{array}{l}\text { 4. Aider à l'investigation (montrer comment visiter et } \\
\text { comment diriger les recherches d'informations, } \\
\text { rappeler les règles du jeu) }\end{array}$ & $\begin{array}{l}\text { 4. Commentaires témoignant des difficultés rencontrées } \\
\text { (demander de l'aide ou exprimer des difficultés) }\end{array}$ \\
\hline $\begin{array}{l}\text { 5. Aider à la lecture et à l'écriture (lire ou épeler à voix } \\
\text { haute) }\end{array}$ & 5. Lire à voix haute ou reformuler les textes \\
\hline 6. Exprimer son approbation & 6. Exprimer son accord \\
\hline 7. Exprimer son désaccord, mettre en doute & 7. Exprimer son doute ou son désaccord \\
\hline $\begin{array}{l}\text { 8. Faire prendre du recul par une interprétation } \\
\text { ponctuelle (susciter la réflexion) }\end{array}$ & $\begin{array}{l}\text { 8. Établir des relations (en lien avec l'expérience } \\
\text { personnelle ou entre plusieurs objets des collections } \\
\text { et portant sur des caractéristiques visibles des objets) }\end{array}$ \\
\hline $\begin{array}{l}\text { 9. Stimuler l'élaboration d'un sens (expliquer ou aider } \\
\text { à trouver le sens ou la fonction d'un objet) }\end{array}$ & $\begin{array}{l}\text { 9. Expliciter le sens ou la fonction des objets } \\
\text { (commentaires en lien avec l'expérience personnelle } \\
\text { ou des connaissances préalables et portant sur } \\
\text { la fonction ou le sens des objets) }\end{array}$ \\
\hline 10. Faire respecter les règles de conduite & $\begin{array}{l}\text { 10. Commentaires témoignant de l'ennui, } \\
\text { de la lassitude ou de la dissipation de l'enfant }\end{array}$ \\
\hline $\begin{array}{l}\text { 11. Orienter et prendre des décisions d'ordre matériel } \\
\text { (interventions organisationnelles) }\end{array}$ & $\begin{array}{l}\text { 11. Commenter son comportement, émettre des décisions } \\
\text { ou suggestions liées à l'organisation et à l'orientation } \\
\text { dans le musée (interventions verbales ou non verbales } \\
\text { comportementales et organisationnelles) }\end{array}$ \\
\hline $\begin{array}{l}\text { 12. Communiquer sur le cadrage secondaire du jeu } \\
\text { (touchant au caractère fictif de la narration, ou au } \\
\text { caractère factice des éléments du jeu) }\end{array}$ & 12. Communiquer sur le cadre secondaire du jeu \\
\hline 13. Exprimer une appréciation ou ses sentiments & 13. Exprimer une appréciation ou ses sentiments \\
\hline
\end{tabular}

Catégorisation des types d'interventions émises au sein des groupes familiaux

d'aide fréquentes par les enfants mettent en lumière des dysfonctionnements du jeu.

\section{Évaluer les apprentissages à l'issue de la visite} Les deux jeux concernés par l'étude ont comme projet d'initier de jeunes enfants à la manière dont on visite un musée dont ils sont encore peu familiers. Les deux jeux ont également pour objectif de développer l'esprit d'observation des enfants. Des questionnaires adaptés à ce type de public ont donc été mis au point pour évaluer la socialisation des enfants au musée, la mémorisation visuelle des œuvres et la compréhension par les enfants de la thématique du musée.

Les questionnaires ont été adaptés aux enfants en privilégiant les épreuves de rappel et de reconnaissance des objets des collections et des éléments du jeu. La conception des questionnaires s'est inspirée de la méthode utilisée par Sylvie Romano au Préau 


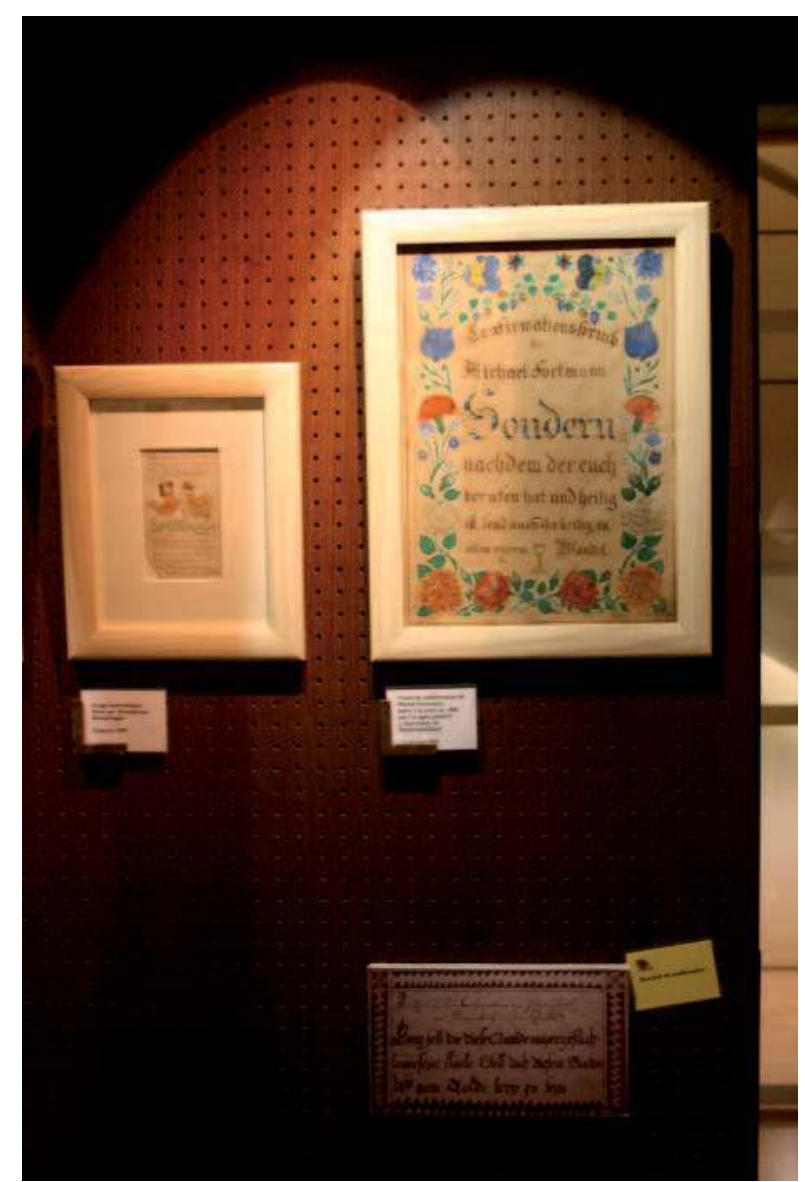

Le souvenir de confirmation accroché en bas de la cimaise est une reproduction factice à l'usage du jeu La vie mystérieuse de l'Oncle Hans. Ce fac-similé est placé à hauteur des enfants et signalé par un cartel de couleur jaune. Le but du jeu consiste à retrouver des images afin de compléter dans un livret-jeu les informations manquantes sur les étapes de la vie d'un personnage fictif I'Oncle Hans.

(c) Claire Barbieux

des Accoules à Marseille (Romano, 1993 : 3-10) ainsi que de celle développée par Hana Gottesdiener et Jean Vilatte pour évaluer les effets d'un livret-jeu sur des enfants de 6 à 14 ans en visite familiale au musée des Beaux-Arts de Quimper (Gottesdiener, Vilatte, 2001 : 167-176). Dans le but d'évaluer les apprentissages perceptivo-cognitifs, ces deux études recouraient à des photographies prises dans le musée comme supports des entretiens avec les enfants. Les photographies présentent des reproductions des lieux et d'objets de l'exposition. À ces photographies sont mêlés autant que de besoin des éléments intrus, photographiés ailleurs mais proches de ceux présents in situ. Ce sont des «distracteurs » qui permettent de contrôler la qualité des réponses et d'éliminer celles faites au hasard.

La reconnaissance des lieux par les enfants, les comportements à adopter dans le musée et la fonction du musée selon les enfants font aussi partie des thèmes explorés par les questionnaires. Ces trois thèmes sont exprimés sous la forme de questions fermées auxquelles les enfants ne pouvaient répondre que par oui ou par non. Les questions sur la reconnaissance des lieux sont illustrées par des photographies représentant des lieux intrus et le musée concerné. La série de questions sur les comportements à adopter au sein du musée interroge les enfants sur ce qu'ils peuvent faire ou ne pas faire dans le musée. Pour cinq des comportements (observer, toucher, lire, apprendre, écrire), en cas de réponse positive de la part de l'enfant, la question embraye ensuite sur son expérience particulière de l'aprèsmidi passée dans le musée.

Enfin, d'autres questions explorent les connaissances des enfants à propos de la thématique du musée abordée à travers le jeu. Il s'agit des images populaires pour l'un et d'une série d'uniformes militaires de la guerre de 1870 pour l'autre. Nous avons interrogé les enfants sur des thèmes pas très évidents : situation de la période historique, noms des soldats ou des images populaires, lien entre les images populaires et les différentes étapes de la vie.... En effet, tel est bien le contenu du discours d'exposition proposé par les deux musées. Par exemple, le livret-jeu du musée de l'Image populaire à Pfaffenhoffen fait appel à une trame narrative fictive autour d'un personnage imaginaire : l'Oncle Hans. Une question supplémentaire a donc vérifié la perception du second degré du jeu par les enfants.

\section{Conclusion : qualités et limites de la méthode utilisée}

Ces instruments de recherche présentent la particularité d'avoir adapté des méthodes étudiant l'apprentissage dans les musées à partir des interventions verbales à la problématique du jeu. Nous avons également conçu des questionnaires complémentaires pour évaluer d'une part la socialisation au musée, et d'autre part la reconnaissance et la compréhension des ouvres vues au cours du jeu par les enfants. L'association de ces deux outils de recherche ne se limite pas à vérifier si oui ou non des objectifs sont atteints, mais cherche aussi à saisir l'expérience ludique des enfants à travers la nature de la communication échangée au cours du jeu. Le test puis la mise en œuvre de ces outils dans le cadre de l'étude des effets d'un livret-jeu et d'un jeu d'observation nous ont paru approcher au plus près du jeu (en tant que « jouer ») des jeunes visiteurs.

Notre étude nous a permis de tirer un bilan qualitatif de l'expérience des jeunes visiteurs lors de la pratique familiale de deux jeux : un jeu d'observation 


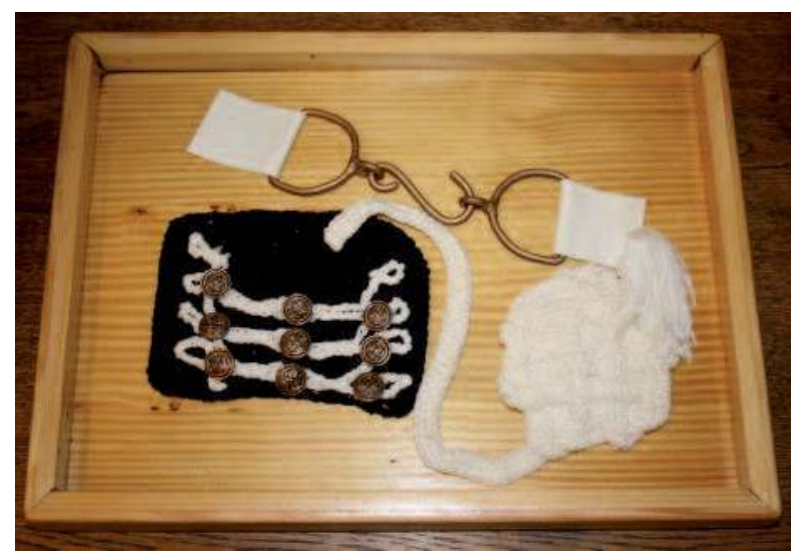

Support tactile de jeu À qui est cet uniforme ? du musée de la Bataille du 6 août 1870 comportant des indices évoquant des éléments d'uniforme de la guerre de 1870 : les enfants doivent retrouver dans le musée quel soldat portait ces éléments caractéristiques sur son uniforme. (c) Claire Barbieux

au musée de la Bataille du 6 août 1870 de Woerth et un livret-jeu au musée de l'Image populaire à Pfaffenhoffen. Dans les deux cas, les jeux semblent avoir contribué à la socialisation des enfants au musée et à leur appropriation de cet espace. Les enfants ont pleinement conscience des comportements spécifiques à adopter au sein du musée. Ils ont appris à utiliser l'espace du musée au cours du jeu et savent où ils peuvent lire et s'informer sur les objets du musée.

Le jeu d'observation À qui est cet uniforme? du musée de la Bataille de 1870 est une réussite en ce qui concerne la mémorisation visuelle des uniformes de la guerre de 1870 sur lesquels porte le jeu. L'étude des interactions des groupes familiaux observés a également révélé que les enfants ont éprouvé un plaisir certain à travers cette activité. La pratique du jeu en famille paraît donc favoriser la découverte du patrimoine culturel par les interactions nombreuses qu'il suscite au sein de la famille.

En ce qui concerne le livret-jeu La vie mystérieuse de l'Oncle Hans du musée de l'Image populaire, les enfants se sont familiarisés avec les images populaires, puisqu'ils sont capables de discerner une image populaire d'un tableau expressionniste, d'un collage cubiste, ou d'une estampe japonaise. Ils ont également bien mémorisé les images du livret-jeu ou celles reproduites sur les fac-similés dans le musée. Les résultats concernant la compréhension du sens des images du jeu sont en revanche plus mitigés. Tout d'abord, les enfants ne perçoivent pas le cadre secondaire du jeu et l'assimilent à la réalité : le personnage fictif du jeu, l'Oncle Hans, a réellement existé pour les enfants. D'autre part, la perception du lien entre les images du jeu et les grandes étapes de la vie est inégale en fonction des différentes images. De plus, le jeu n'offre pas une mise en contexte suffisante pour comprendre les liens entre les images et les rites de passage religieux : baptême et confirmation.

La recherche d'informations et le remplissage du texte à trous du livret-jeu ne semblent donc pas apporter un apprentissage supplémentaire en terme de compréhension du contenu des collections par rapport à un jeu uniquement basé sur l'observation. La pratique d'un texte à trous au musée engendre des difficultés dont on ne mesure bien souvent pas assez l'importance au niveau de l'écriture et de la lecture. Une part importante des interactions familiales sont destinées à aider à lire ou à écrire $(26 \%$ des interventions de coéducation) ou à exprimer des difficultés ou demander de l'aide ( $14 \%$ des interventions des enfants). L'expérience s'avère aussi moins satisfaisante au niveau du plaisir et de la motivation des enfants. Au regard des efforts déployés par les familles autour de la lecture et de l'écriture, les résultats en terme de compréhension des images populaires paraissent un peu faibles.

Nous avons souhaité mettre en œuvre une méthode pluri-technique, ce qui est évidemment plus chronophage qu'un entretien ou un questionnaire seul à l'issue de la visite. De plus, ces deux outils d'analyse complémentaires contiennent en eux-mêmes des limites influant sur la fiabilité des résultats qu'ils produisent.

Le recours à la vidéo permet une certaine exhaustivité des observations des comportements et des remarques spontanées des visiteurs. Durant la pratique du jeu, l'observation filmée s'est toujours faite en retrait pour ne pas gêner les déplacements des familles ou les interactions entre leurs membres. Le rapport des visiteurs à la caméra est un peu plus malaisé dans le cas de groupes restreints (dyade parent-enfant), ainsi qu'en début de visite. Toutefois, ce facteur d'intimidation s'estompe rapidement du fait de la convivialité et de la participation active des enfants impliquées par le jeu. L'enregistrement filmé pouvant difficilement se faire à l'insu des visiteurs, les familles étaient averties à l'avance du protocole d'évaluation, ce qui induit une certaine artificialité de la situation. Certains participants ont parfois pris l'observateur à témoin de leurs difficultés ou lui ont fait part de leurs impressions, mais les contacts n'ont jamais été établis à son initiative.

En ce qui concerne les questionnaires post-visite, ceux-ci évaluent la socialisation des enfants au musée, mais aussi les apprentissages cognitifs définis 
au préalable dans les objectifs à partir d'un test de reconnaissance à l'issue du jeu. Cependant, la seule vérification de connaissances retenues à l'issue de la visite ne peut prétendre mettre au jour l'ensemble de ce que les visiteurs se sont appropriés durant le jeu.

Des améliorations pourraient être également apportées. Les données socioculturelles sont connues pour influencer les pratiques culturelles. Le genre, l'âge ou la fréquence de visite des musées qui peuvent être facilement recueillis dans le cadre du questionnaire post-visite pourraient être croisés avec les comportements et les interactions observés au cours de la visite.

L'échantillon réduit sur lequel porte ces recherches ne permet pas de donner une portée générale à ces résultats. Toutefois, cette recherche appliquée à deux jeux permet de soulever la question du choix d'une mise en forme ludique plutôt qu'une autre lors de la conception de jeux pour les enfants dans les musées. La forme si répandue du livret-jeu (du fait de sa simplicité de conception et de son faible coût) permet certes de focaliser l'attention des enfants, mais peuton réellement qualifier de jeu, une activité si le plaisir paraît faible et si les enfants rencontrent de nombreuses difficultés lors de l'exercice de remplissage du livret? La recherche d'informations factuelles ne paraît pas garantir à elle seule une bonne compréhension des enfants.

Il serait nécessaire de recueillir les interventions verbales et non verbales de familles plus nombreuses et d'interroger un plus grand nombre d'enfants afin de valider ou pondérer les résultats obtenus ici. Il serait également intéressant d'étudier les effets d'autres types de jeux proposés dans les musées pour permettre aux professionnels des musées d'élaborer des jeux de manière plus éclairée.

\section{Bibliographie}

Bateson, G. Une théorie du jeu et du fantasme, in Vers une écologie de l'esprit. Paris : Le Seuil, 1977, pp. 209-224.

Borun, M., Chambers, M. et Cleghorn, A. Families are learning in science museums, Curator, vol. 39, n², 1996, pp. 123-138.

Bougère, G. Jouer/Apprendre. Paris : Économica, 2005.

Bruner, J. Le développement de l'enfant; savoir faire, savoir dire. Paris, PUF, 1983, pp. 52-53.
Chauvier, S. Qu'est-ce qu'un jeu ? Paris : Vrin, 2007.

Eidelman, J. et Van Praët, M. La muséologie des sciences et ses publics. Regards croisés sur la Grande Galerie de l'évolution du Muséum national d'Histoire naturelle. Paris : Presses universitaires de France, 2000.

Csikszentmihalyi, M. et Robinson, R.-E. Beyond Boredom and Anxiety. San Francisco : Jossey-Bass, 1975.

Duflos, C. Jouer et philosopher. Paris : Presses universitaires de France, 1997.

Dufresne-Tassé, C. Informations nécessaires à la conception d'une mise en exposition des phénomènes scientifiques, Publics et Musées, nº7, 1995, pp. 25-46.

Goffman, E. Les cadres de l'expérience. Paris : Éditons de Minuit, 1991.

Gottesdiener, H. et Vilatte, J. Impact of a game booklet on family visit to an art exhibition, Empirical Studies of the Arts, vol. 19, 2001, pp. 167-176.

Henriot, J. Sous couleur de jouer : la métaphore du ludique. Paris : José Corti, 1989.

Jonchéry, A. Se rendre au musée en famille, la Lettre de l'OCIM, $\mathrm{n}^{\circ} 115$, 2008, pp. 4-14.

Mead, G.-H. L'esprit, le soi et la société. Paris : PUF, 1964.

Niquette, M. Éléments critiques pour l'analyse de la réception et du partage social des connaissances, Publics et Musées, n5, 1994, pp. 79-97.

Piaget, J. La formation du symbole chez l'enfant. Paris : Delachaux et Niestlé, 1978.

Power, T.-G. Play Exploration and learning in children and animals. Mahwah : Lawrence Erlbaum Associates, 2000.

Romano, S. Entre musées et enfants de 4 à 6 ans, une action utile ou non ?, la Lettre de l'OCIM, n60, 1998, pp. 3-9.

Vygostky, L.-S. Play and its role in the mental development of child, Soviet Psychology, vol. 5, n³, 1967, pp. 6-18. 